\title{
Another approach to the automorphism theorem for Teichmüller spaces
}

\author{
Ege Fujikawa
}

\begin{abstract}
It has been proved that for a Riemann surface of non-exceptional type, every biholomorphic automorphism of the Teichmüller space is induced by a quasiconformal automorphism of the Riemann surface. The proof is a combination of two theorems. One theorem states that the above statement is true if a Riemann surface has the isometry property, which was proved by Earle and Gardiner and is called the automorphism theorem. Another theorem states that every Riemann surface of non-exceptional type has the isometry property, which was finally proved by Markovic. In this paper, we give another approach to a proof of the automorphism theorem.
\end{abstract}

\section{Introduction}

The Teichmüller space $T(R)$ of a Riemann surface $R$ is the set of all equivalence classes $[f]$ of quasiconformal homeomorphisms $f$ on $R$. We say that two quasiconformal homeomorphisms $f_{1}$ and $f_{2}$ on $R$ are equivalent if there exists a conformal homeomorphism $h: f_{1}(R) \rightarrow f_{2}(R)$ such that $f_{2}^{-1} \circ h \circ f_{1}$ is homotopic to the identity. All homotopies are considered to be relative to the ideal boundary at infinity. A distance between two points $\left[f_{1}\right]$ and $\left[f_{2}\right]$ in $T(R)$ is defined by $d\left(\left[f_{1}\right],\left[f_{2}\right]\right)=(1 / 2) \log K(f)$, where $f$ is an extremal quasiconformal homeomorphism in the sense that its maximal dilatation $K(f)$ is minimal in the homotopy class of $f_{2} \circ f_{1}^{-1}$. Then $d$ is a complete distance on $T(R)$, which is called the Teichmüller distance.

We assume that a Riemann surface $R$ is of hyperbolic type. Namely, it is represented by a quotient space $\mathbb{H}^{+} / \Gamma$ of the upper half-plane $\mathbb{H}^{+}=\{z \in \mathbb{C} \mid$ $\operatorname{Im} z>0\}$ by a torsion free Fuchsian group $\Gamma$. Let $R^{\prime}=\mathbb{H}^{-} / \Gamma$ be the complex conjugate of $R$ where $\mathbb{H}^{-}=\{z \in \mathbb{C} \mid \operatorname{Im} z<0\}$, and $B\left(R^{\prime}\right)$ the complex Banach space of all bounded holomorphic quadratic differentials on $R^{\prime}$ with the hyperbolic supremum norm. Then the Teichmüller space $T(R)$ is a complex Banach manifold modeled on $B\left(R^{\prime}\right)$. In fact, $T(R)$ is embedded in $B\left(R^{\prime}\right)$ as a bounded contractible domain. For details, see [13] and [16].

The quasiconformal mapping class $[g]$ on a Riemann surface $R$ is a homotopy equivalence class of quasiconformal automorphisms $g$ of $R$, and the quasiconformal mapping class group $\mathrm{MCG}(R)$ of $R$ is the set of all quasiconformal mapping classes

2000 Mathematics Subject Classification. Primary 30F60; Secondary 37F30.

Key words and phrases. Riemann surface of infinite type, quasiconformal map, mapping class group, Teichmüller modular group. 
on $R$. Every element $[g] \in \mathrm{MCG}(R)$ induces a biholomorphic automorphism $\rho_{g}$ of $T(R)$ by $[f] \mapsto\left[f \circ g^{-1}\right]$. This automorphism $\rho_{g}$ is also an isometry with respect to the Teichmüller distance. Let $\operatorname{Aut}(T(R))$ be the group of all biholomorphic automorphisms of $T(R)$. Then we have a homomorphism

$$
\iota: \operatorname{MCG}(R) \rightarrow \operatorname{Aut}(T(R))
$$

by $[g] \mapsto \rho_{g}$, and define the Teichmüller modular group by

$$
\operatorname{Mod}(R)=\iota(\operatorname{MCG}(R)) .
$$

It was proved in $[\mathbf{3}],[\mathbf{5}]$ and $[\mathbf{1 5}]$ that $\iota$ is injective (faithful) for all Riemann surfaces $R$ of non-exceptional type, and then we may identify $\operatorname{Mod}(R)$ with $\operatorname{MCG}(R)$. Here we say that a Riemann surface $R$ is of exceptional type if $R$ has finite hyperbolic area and satisfies $2 g+n \leq 4$, where $g$ is the genus of $R$ and $n$ is the number of punctures of $R$.

It was considered whether $\iota$ is surjective, namely whether every biholomorphic automorphism of the Teichmüller space is induced by a quasiconformal automorphism of the Riemann surface. Royden [17] proved that $\iota$ is surjective for all compact Riemann surfaces $R$ of genus $g \geq 2$, and Earle and Kra [4] proved the statement for all non-exceptional analytically finite Riemann surfaces. After that, it was a problem in researches on infinite dimensional Teichmüller spaces whether $\iota$ is surjective for all Riemann surfaces of analytically infinite type. After several partial solutions, finally the following theorem was proved.

TheOREM 1.1. For every Riemann surface $R$ of non-exceptional type, we have

$$
\operatorname{Aut}(T(R))=\operatorname{Mod}(R) \text {. }
$$

Theorem 1.1 is proved by a combination of results by Earle and Gardiner [2] and Markovic [14]. To state their results, we begin by the following definitions. We say that a quadratic differential on a Riemann surface $R$ is integrable if the norm

$$
\|\varphi\|=\iint_{R}|\varphi|
$$

is finite, and let $Q(R)$ be the complex Banach space of integrable holomorphic quadratic differentials on $R$ with the above norm. We say that a Riemann surface $R$ has the isometry property if, for any Riemann surfaces $S_{1}$ and $S_{2}$ that are quasiconformally equivalent to $R$, and for every surjective $\mathbb{C}$-linear isometry $G: Q\left(S_{1}\right) \rightarrow Q\left(S_{2}\right)$, there exist $\theta \in \mathbb{C}$ with $|\theta|=1$ and a conformal homeomorphism $g: S_{1} \rightarrow S_{2}$ such that

$$
G(\varphi)=\theta \cdot(\varphi \circ g)\left(g^{\prime}\right)^{2}
$$

for all $\varphi \in Q\left(S_{2}\right)$.

Earle and Gardiner [2] gives a sufficient condition for Riemann surfaces for which Theorem 1.1 is true.

THEOREM $1.2([2]$ (the automorphism theorem)). Let $R$ be a Riemann surface having the isometry property. Then $\operatorname{Aut}(T(R))=\operatorname{Mod}(R)$.

In the paper [2], they also proved that all Riemann surfaces of topologically finite type have the isometry property. Furthermore, Lakic [12] proved that all Riemann surfaces of finite genus have the isometry property. Finally, Markovic $[\mathbf{1 4}]$ proved the following. 
THEOREM $1.3([\mathbf{1 4}])$. Every Riemann surface of non-exceptional type has the isometry property.

In this paper, we give another approach to a proof of the automorphism theorem. We review the proof by Earle and Gardiner in the next section, and state our proof in Section 3.

\section{Proof of the automorphism theorem by Earle and Gardiner}

In this section, we recall an outline of the proof of the automorphism theorem by Earle and Gardiner [2]. See also Chapter 8 in [11].

Let $E: T(R) \rightarrow T(R)$ be a biholomorphic automorphism of $T(R)$. For a point $p=\left[f_{1}\right] \in T(R)$, set $E(p)=\left[f_{2}\right] \in T(R)$. For each $i=1,2$, the quasiconformal homeomorphism $f_{i}: R \rightarrow S_{i}$ induces a geometric automorphism $\rho_{f_{i}}: T(R) \rightarrow$ $T\left(S_{i}\right)$ that maps $[f] \mapsto\left[f \circ f_{i}^{-1}\right]$. Then $F:=\rho_{f_{2}} \circ E \circ \rho_{f_{1}}^{-1}: T\left(S_{1}\right) \rightarrow T\left(S_{2}\right)$ is a holomorphic isomorphism that preserves the base points.

We fix $i$ and denote $S_{i}$ by $S$. The dual space $Q^{*}(S)$ of $Q(S)$ is identified with the tangent space $T_{0}(T(S))$ of $T(S)$ at the base point. Indeed, let $\operatorname{Belt}(S)$ be the set of all Beltrami differentials $\mu$ on $S$, which have the norm $\|\mu\|=\operatorname{ess} \sup _{z \in S}|\mu(z)|<\infty$, and set $\operatorname{Belt}(S)_{1}=\{\mu \in \operatorname{Belt}(S) \mid\|\mu\|<1\}$. Let $\Phi_{S}: \operatorname{Belt}(S)_{1} \rightarrow T(S)$ be the Bers projection which maps $\mu \mapsto h^{\mu}$, where $h^{\mu}$ is a quasiconformal homeomorphism on $S$ whose Beltrami coefficient is $\mu$. The derivative $\Phi_{S}^{\prime}(0): \operatorname{Belt}(S) \rightarrow T_{0}(T(S))$ of $\Phi_{S}$ at the base point is a surjective homomorphism. Thus $T_{0}(T(S))$ is isomorphic to the quotient space $\operatorname{Belt}(S) / \operatorname{Ker}\left(\Phi_{S}^{\prime}(0)\right)$. On the other hand, let $P: \operatorname{Belt}(S) \rightarrow Q^{*}(S)$ be the surjective linear map defined by

$$
P \mu(\varphi)=\iint_{S} \mu \varphi
$$

for $\mu \in \operatorname{Belt}(S)$ and for $\varphi \in Q(S)$. Since $\operatorname{Ker} P=\operatorname{Ker}\left(\Phi_{S}^{\prime}(0)\right)$ (see [6]), we see that $Q^{*}(S)$ is isomorphic to $\operatorname{Belt}(S) / \operatorname{Ker}\left(\Phi_{S}^{\prime}(0)\right)$. Then the map $\Phi_{S}^{\prime}(0) \mu \mapsto P \mu$ gives an isomorphism between $T_{0}(T(S))$ and $Q^{*}(S)$. The tangent space $T_{0}(T(S))$ of $T(S)$ at the base point has the Teichmüller norm

$$
\left\|\Phi_{S}^{\prime}(0) \mu\right\|=\lim _{t \rightarrow+0} \frac{d\left(\Phi_{S}(0), \Phi_{S}(t \mu)\right)}{t} .
$$

By the above identification, the Teichmüller norm coincides with the standard dual norm on $Q^{*}(S)$.

We see that $F^{\prime}([i d])$ is a $\mathbb{C}$-linear isometry of $Q^{*}\left(S_{1}\right)$ onto $Q^{*}\left(S_{2}\right)$. Indeed, since $F$ is biholomorphic, $F^{\prime}([i d])$ is an invertible $\mathbb{C}$-linear map. Furthermore it preserves the Teichmüller norm. This follows from the fact that the biholomorphic isomorphism $F$ preserves the Kobayashi distance, and that the Kobayashi distance coincides with the Teichmüller distance (see $[\mathbf{9}]$ ).

The following proposition states that $F^{\prime}([i d])$ induces a $\mathbb{C}$-linear isometry of $Q\left(S_{2}\right)$ onto $Q\left(S_{1}\right)$. Note that $Q\left(S_{i}\right)$ is not reflexive if it is infinite dimensional.

Proposition 2.1 (the adjointness theorem). Let $S_{1}$ and $S_{2}$ be Riemann surfaces and $I: Q^{*}\left(S_{1}\right) \rightarrow Q^{*}\left(S_{2}\right)$ a $\mathbb{C}$-linear isometry. Then there exists a $\mathbb{C}$-linear isometry $G: Q\left(S_{2}\right) \rightarrow Q\left(S_{1}\right)$ such that $G^{*}=I$. 
By applying the adjointness theorem to our case, there exists a $\mathbb{C}$-linear isometry $G: Q\left(S_{2}\right) \rightarrow Q\left(S_{1}\right)$ such that $F^{\prime}([i d])=G^{*}$. Since $R$ has the isometry property, there exist $\theta \in \mathbb{C}$ with $|\theta|=1$ and a conformal homeomorphism $g: S_{1} \rightarrow S_{2}$ such that $G(\varphi)=\theta \cdot(\varphi \circ g)\left(g^{\prime}\right)^{2}$ for all $\varphi \in Q\left(S_{2}\right)$. Then $g$ induces a geometric isomorphism $\rho_{g}: T\left(S_{1}\right) \rightarrow T\left(S_{2}\right)$, which preserves the base points.

Set $\omega_{p}:=\left(\rho_{f_{2}}\right)^{-1} \circ \rho_{g} \circ \rho_{f_{1}}=\rho_{f_{2}-1} \circ g \circ f_{1}: T(R) \rightarrow T(R)$, which is an element of $\operatorname{Mod}(R)$. Then $\omega_{p}(p)=E(p)$. To complete a proof, we show that $\omega_{p}$ is independent of $p$, namely $\omega_{p}=E$ on $T(R)$.

Proposition 2.2 (the uniqueness theorem). Let $R$ be a Riemann surface having the isometry property, and $H: T(R) \rightarrow T(R)$ a holomorphic automorphism satisfying $H(q)=q$ and $H^{\prime}(q)=\theta \cdot i d(\theta \in \mathbb{C})$ for some $q \in T(R)$. Then $H$ is the identity.

We would like to apply the uniqueness theorem to $H=\omega_{p} \circ E^{-1}$. It is clear that $\omega_{p} \circ E^{-1}(p)=p$. By another consideration, we see that $\rho_{g}^{\prime}([i d]): Q^{*}\left(S_{1}\right) \rightarrow Q^{*}\left(S_{2}\right)$ is the adjoint of $\theta^{-1} G$ (see Lemma 4.2 in $[\mathbf{2}]$ ), and thus $F^{\prime}([i d])=\theta \rho_{g}^{\prime}([i d])$. Then we have

$$
E^{\prime}(p)=\left(\left(\rho_{f_{2}}\right)^{-1} \circ F \circ \rho_{f_{1}}\right)^{\prime}(p)=\theta\left(\left(\rho_{f_{2}}\right)^{-1} \circ \rho_{g} \circ \rho_{f_{1}}\right)^{\prime}(p)=\theta \omega_{p}^{\prime}(p),
$$

where the derivatives are at the base point. Hence we have $\left(\omega_{p} \circ E^{-1}\right)^{\prime}(p)=\theta \cdot i d$. Then by the uniqueness theorem, we conclude that $\omega_{p}=E$ on $T(R)$.

\section{Main theorem and proof}

In the proof of the automorphism theorem by Earle and Gardiner, the uniqueness theorem was used to conclude that $\omega_{p}=E$ on $T(R)$. The purpose of this paper is to give another proof of the automorphism theorem by considering the discontinuity of Teichmüller modular groups, instead of using the uniqueness theorem. However we have to assume that Riemann surfaces have the bounded geometry.

We say that a Riemann surface $R$ has the bounded geometry if $R$ satisfies the following three conditions:

(i) the lower bound condition: the injectivity radius at any point of $R$ except cusp neighborhoods are uniformly bounded away from zero.

(ii) the upper bound condition: there exists a subdomain $R^{*}$ of $R$ such that the injectivity radius at any point of $R^{*}$ is uniformly bounded from above and that the simple closed curves in $R^{*}$ carry the fundamental group of $R$.

(iii) $R$ has no ideal boundary at infinity, namely the Fuchsian model of $R$ is of the first kind.

The bounded geometry condition is quasiconformally invariant, and every nonuniversal normal cover of a Riemann surface of analytically finite type has the bounded geometry. Our theorem is the following.

THEOREM 3.1. Let $R$ be a Riemann surface that is of non-exceptional type and has the bounded geometry, and $\Psi$ a biholomorphic automorphism of $T(R)$. Suppose that, for every point $p \in T(R)$, there exists an element $\omega_{p} \in \operatorname{Mod}(R)$ such that $\Psi(p)=\omega_{p}(p)$. Then $\Psi \in \operatorname{Mod}(R)$.

We say that the Teichmüller modular group $\operatorname{Mod}(R)$ acts at a point $p \in T(R)$ discontinuously if there exists a neighborhood $U$ of $p$ such that $\chi(U) \cap U=\emptyset$ for all 
$\chi \in \operatorname{Mod}(R)-\operatorname{Stab}(p)$ and that the stabilizer subgroup $\operatorname{Stab}(p)=\{\chi \in \operatorname{Mod}(R) \mid$ $\chi(p)=p\}$ consists of finitely many elements. Set

$$
\Omega(\operatorname{Mod}(R))=\{p \in T(R) \mid \operatorname{Mod}(R) \text { acts at } p \text { discontinuously }\} .
$$

For a Riemann surface $R$ of analytically finite type, the Teichmüller modular group $\operatorname{Mod}(R)$ acts on $T(R)$ discontinuously, namely $\Omega(\operatorname{Mod}(R))=T(R)$ (see Section 8 in $[\mathbf{1 0}])$. However, for a Riemann surface of analytically infinite type, the action of $\operatorname{Mod}(R)$ is not discontinuous, in general. In a series of our papers [6], [7] and $[\mathbf{8}]$, we studied the discontinuity of Teichmüller modular groups, and proved the following.

Proposition $3.2([\mathbf{6}])$. If a Riemann surface $R$ is of non-exceptional type and has the bounded geometry, then $\Omega(\operatorname{Mod}(R)) \neq \emptyset$.

We will prove Theorem 3.1 by using Proposition 3.2.

Proof of Theorem 3.1. By Proposition 3.2, we take a point $p \in \Omega(\operatorname{Mod}(R))$. Then there exists an open ball $U_{\delta}$ centered at $p$ with radius $\delta>0$ such that $\chi\left(U_{\delta}\right) \cap U_{\delta}=\emptyset$ for all elements $\chi \in \operatorname{Mod}(R)-\operatorname{Stab}(p)$, where $\operatorname{Stab}(p)$ consists of finitely many elements. Since the action of $\operatorname{Mod}(R)$ is isometric and any points $p$ and $p^{\prime}$ in $T(R)$ can be joined by a segment of length $d\left(p, p^{\prime}\right)$, we have $d(p, \chi(p)) \geq 2 \delta$ for all $\chi \in \operatorname{Mod}(R)-\operatorname{Stab}(p)$. We take an arbitrary point $q \in U_{\delta / 2}$, namely $d(p, q)<\delta / 2$. Then $d(\chi(p), \chi(q))<\delta / 2$ for all $\chi \in \operatorname{Mod}(R)$. Thus $d(q, \chi(q))>\delta$ for all $\chi \in \operatorname{Mod}(R)-\operatorname{Stab}(p)$. For every point $q \in U_{\delta / 2}$,

$$
\begin{aligned}
d\left(q, \omega_{p}^{-1} \circ \omega_{q}(q)\right) & =d\left(\omega_{p}(q), \omega_{q}(q)\right) \\
& \leq d\left(\omega_{p}(q), \omega_{p}(p)\right)+d\left(\omega_{p}(p), \omega_{q}(q)\right) \\
& =d(p, q)+d(\Psi(p), \Psi(q)) \\
& =2 d(p, q)<\delta
\end{aligned}
$$

by the fact that the biholomorphic automorphism $\Psi$ preserves the Teichmüller distance. Thus $\omega_{p}^{-1} \circ \omega_{q} \in \operatorname{Stab}(p)$ for every $q \in U_{\delta / 2}$. We denote the finitely many elements of $\operatorname{Stab}(p)$ by $\left\{\chi_{i}\right\}_{i=1}^{n}$, and set

$$
Q_{i}=\left\{q \in U_{\delta / 2} \mid \omega_{p}^{-1} \circ \omega_{q}(q)=\chi_{i}(q)\right\}=\left\{q \in U_{\delta / 2} \mid \Psi(q)=\omega_{p} \circ \chi_{i}(q)\right\} .
$$

Then $U_{\delta / 2}=\bigcup_{i=1}^{n} Q_{i}$. Since all $Q_{i}$ are closed in $U_{\delta / 2}$, at least one of them, say $Q_{1}$, has an interior point. For all points $q \in Q_{1}$, we have $\Psi(q)=\omega_{p} \circ \chi_{1}(q)$. Since $T(R)$ is connected, the uniqueness theorem for holomorphic functions implies that $\Psi=\omega_{p} \circ \chi_{1}$ on $T(R)$. Thus $\Psi \in \operatorname{Mod}(R)$.

Theorem 3.1 with its proof gives another approach to the automorphism theorem. This proof is a natural generalization of the proof for finite dimensional cases in $[\mathbf{4}]$ and $[\mathbf{1 7}]$.

In Theorem 3.1, we use the assumption that $R$ has the bounded geometry to apply Proposition 3.2. Thus Theorem 3.1 with its proof is true for all Riemann surfaces $R$ satisfying $\Omega(\operatorname{Mod}(R)) \neq \emptyset$. We have an example of a Riemann surface $R$ such that $R$ does not satisfy the upper bound condition but satisfies $\Omega(\operatorname{Mod}(R)) \neq \emptyset$. On the other hand, if $R$ does not satisfy the lower bound condition, then $\Omega(\operatorname{Mod}(R))=\emptyset$ (see Theorem 1 in $[6])$. We conjecture that $\Omega(\operatorname{Mod}(R)) \neq \emptyset$ if and only if $R$ satisfies the lower bound condition and has no ideal boundary at infinity, and our proof of the automorphism theorem is valid for all such Riemann surfaces. 


\section{References}

[1] C. J. Earle, Teichmüller theory, in Discrete Groups and Automorphic Functions, 143-162, Academic Press, London - New York - San Francisco, 1977.

[2] C. J. Earle and F. P. Gardiner, Geometric isomorphisms between infinite dimensional Teichmüller spaces, Trans. Amer. Math. Soc. 348 (1996), 1163-1190.

[3] C. J. Earle, F. P. Gardiner and N. Lakic, Teichmüller spaces with asymptotic conformal equivalence, preprint.

[4] C. J. Earle and I. Kra, On isometries between Teichmüller spaces, Duke. Math. J. 41 (1974), 583-591.

[5] A. L. Epstein, Effectiveness of Teichmüller modular groups, In the tradition of Ahlfors and Bers, Contemporary Math. 256 (2000), 69-74.

[6] E. Fujikawa, Limit sets and regions of discontinuity of Teichmüller modular groups, Proc. Amer. Math. Soc. 132 (2004), 117-126.

[7] E. Fujikawa, Modular groups acting on infinite dimensional Teichmüller spaces, In the tradition of Ahlfors and Bers III, Contemporary Math. 355 (2004), 239-253.

[8] E. Fujikawa, H. Shiga and M. Taniguchi, On the action of the mapping class group for Riemann surfaces of infinite type, J. Math. Soc. Japan 56 (2004), 1069-1086.

[9] F. P. Gardiner, Approximation of infinite dimensional Teichmüller Spaces, Trans. Amer. Math. Soc. 282 (1984), 367-383.

[10] F. P. Gardiner, Teichmüller theory and quadratic differentials, Wiley-Interscience, New York 1987.

[11] F. P. Gardiner and N. Lakic, Quasiconformal Teichmüller Theory, Mathematical Surveys and Monographs 76, Amer. Math. Soc., 2000.

[12] N. Lakic, An isometry theorem for quadratic differentials on Riemann surface of finite type, Trans. Amer, Math. Soc. 394 (1997), 2951-2967.

[13] O. Lehto, Univalent Functions and Teichmüller Spaces, Graduate Text in Math. 109, Springer.

[14] V. Markovic, Biholomorphic maps between Teichmüller spaces, Duke Math. J. 120 (2003), 405-431.

[15] K. Matsuzaki, Inclusion relations between the Bers embeddings of Teichmüller spaces, Israel J. Math. 140 (2004) 113-124.

[16] S. Nag, The Complex Analytic Theory of Teichmüller Spaces, John Wiley \& Sons 1988.

[17] H. L. Royden, Automorphisms and isometries of Teichmüller spaces, Ann. Math. Stud. 66 (1970), 369-384.

Department of Mathematical and Computing Sciences, Tokyo Institute of TechNOlOGy, OH-OKayama, Meguro-ku, TOKYo, 152-8552 Japan

Current address: Department of Mathematics, Sophia University, 7-1 Kioi-cho, Chiyoda-ku, Tokyo, 102-8554 Japan

E-mail address: fujikawa@mm.sophia.ac.jp 